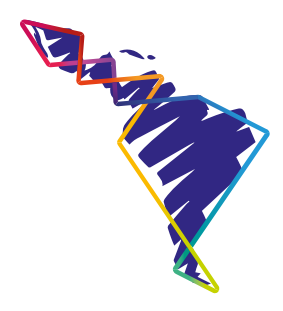

\title{
Vicisitudes en la presencialidad remota de los estudiantes de la carrera Gestión Empresarial del Turismo Sostenible del Campus Pérez Zeledón, Sede Regional Brunca, Universidad Nacional
}

Vicissitudes in the remote presence of the students of the Business Management of Sustainable Tourism program at the Pérez Zeledón Campus, Brunca Regional Headquarters, Universidad Nacional

\author{
Vicissitudes na presença remota \\ dos alunos da carreira de Gestão \\ Empresarial do Turismo Sustentável no \\ Campus Pérez Zeledón, Sede Regional \\ de Brunca, Universidad Nacional
}

M BA. Erick Roberto Madrigal Villanueva ${ }^{1}$ Licda. Yoselyn Pamela Ugalde Jiménez ${ }^{2}$

Recibido: 18-5-2021 - Aceptado: 5-10-2021

1 Erick Roberto Madrigal Villanueva, Maestría en Mercadeo Empresarial, licenciado en Docencia, bachiller en Gestión Empresarial del Turismo Sostenible, docente en la Universidad Nacional, Sede Regional Brunca, Costa Rica. Correo electrónico: erick.madrigal.villanueva@una.ac.cr. (iD https://orcid.org/0000-0003-4317-3422

2 Yoselyn Pamela Ugalde Jiménez, licenciada en Derecho, notaria, bachiller en Gestión Empresarial del Turismo Sostenible, asesora legal del Instituto de Desarrollo Rural, Costa Rica. Correo electrónico: yugalde@ inder.go.cr. (D) https://orcid.org/0000-0002-5801-9690 


\title{
Resumen
}

La presencialidad remota (virtualidad) trae una sumatoria de retos, inconvenientes, dificultades, desplazamientos y procesos de adaptación para alumnos, docentes y el sistema educativo. El objetivo del artículo es hacer visibles las diferentes vicisitudes que los estudiantes de tercer y cuarto año de la carrera Gestión Empresarial del Turismo Sostenible del Campus Pérez Zeledón, Sede Regional Brunca de la Universidad Nacional, han enfrentado a lo largo de más de un año en presencialidad remota. Esos estudiantes son, en su mayoría, de la zona rural de la Región Brunca y Pacífico Central; además, tenían ya un modelo de enseñanza y aprendizaje establecido y que de pronto cambia por la virtualidad, la tecnología, la comunicación a distancia, sin hacer ningún tipo de excepción en el nivel de conectividad o acceso a Internet. Esta investigación es de carácter cualitativo descriptivo, ya que se conoce las apreciaciones de cada uno de los participantes, a través del instrumento de encuesta; a partir de esta, se realizan comparaciones respecto a otros estudios llevados a cabo por la universidad con toda la población estudiantil. Algunos de los resultados encontrados han sido retos e inconvenientes en conectividad, sumados a una mayor carga de trabajos e incluso a la aceptación o percepción del tipo de clases que se reciben. Por último, esta indagación concluye que la virtualidad y la presencialidad, modalidades que han impactado la forma de enseñanza y aprendizaje, han revolucionado los sistemas educativos, modificando un sinnúmero de conductas y metodologías, con la finalidad de lograr el objetivo planteado: terminar la carrera universitaria.

Palabras clave: aprendizaje en línea, aprendizaje a distancia, educación universitaria, educación a distancia, tecnología educacional.

\begin{abstract}
The remote presence (Virtuality) brings a sum of challenges, inconveniences, difficulties, displacements and adaptation processes for students, teachers and the educational system. The objective of the article is to make visible the different vicissitudes that third and fourth year students of the Business Management of Sustainable Tourism program at the Pérez Zeledón Campus, Brunca Regional Headquarters of the Universidad Nacional, have faced over more than a year under the modality of remote presence. Students, mostly from the rural area of the Brunca and Central Pacific Region, who already had an established teaching and learning model, suddenly were exposed to changes due to virtuality, technology, distant communication, without there being any kind of exception at the level of connectivity or access to the internet. This research is qualitative and descriptive, since the appreciations of each of the students are known through the survey instrument and from there comparisons are made with respect to other studies carried out by the university to the entire student population. Some of the results found have been challenges and inconveniences in connectivity, added to a greater workload and even to the acceptance or perception of the type of classes they receive. Finally, this research concludes on how virtuality and in-person modalities have impacted the way of teaching and learning, and revolutionized educational systems, modifying a number of behaviors and methodologies in order to achieve the proposed objective: to obtain a university degree.
\end{abstract}


Keywords: Online learning, Distance learning, University education, Distance education, educational technology.

\section{Resumo}

A presença remota (Virtualidade) traz um somatório de desafios, incômodos, dificuldades, deslocamentos e processos de adaptação para alunos, professores e sistema educacional. O objetivo do artigo é tornar visíveis as diferentes vicissitudes que os alunos do terceiro e quarto ano da carreira de Gestão Empresarial do Turismo Sustentável do Campus Pérez Zeledón, Sede Regional de Brunca da Universidad Nacional, enfrentaram ao longo de mais de um ano em presença remota. Estudantes, maioritariamente da zona rural de Brunca e da Região do Pacífico Centro, que também já tinham um modelo de ensino e aprendizagem estabelecido que muda repentinamente devido à virtualidade, tecnologia, comunicação à distância, sem fazer qualquer tipo de exceção ao nível da conectividade ou do acesso à Internet. A pesquisa é qualitativa e descritiva, uma vez que as apreciações de cada um dos alunos são conhecidas por meio do instrumento de pesquisa e a partir dela são feitas comparações com outros estudos realizados pela universidade para toda a população discente. Alguns dos resultados encontrados têm sido desafios e inconvenientes na conectividade, somados a uma maior carga horária e até mesmo à aceitação ou percepção do tipo de aulas que recebem. Por fim, esta pesquisa conclui sobre como a virtualidade e a presencialidade, modalidades que têm impactado o modo de ensinar e aprender, revolucionaram os sistemas educacionais, modificando uma série de comportamentos e metodologias para atingir o objetivo proposto: terminar o curso superior.

Palavras-chave: Educação online, Educação a distância, Educação universitária, Educação a distância, tecnologia educacional.

\section{Introducción y antecentes}

En el 2020, específicamente en marzo, se registró el primer caso de Covid-19 en Costa Rica y, con esto, se tomó en el país una serie de medidas de prevención. Estas cambiaron, de una manera quizá drástica, los modelos y metodologías de desarrollo que por muchos años se han llevado a cabo en todo tipo de industria y actividades de la sociedad en general.

La educación no fue la excepción del cambio necesario y obligatorio ante la crisis sanitaria generada, por tanto, fue acertado implementar un plan de contingencia emitido por la Rectoría de la Universidad Nacional, el 20 de marzo del 2020, para la "Modificación de las actividades académicas durante la situación de emergencia por COVID-19 en la Universidad Nacional" (circular UNA-VD-DIS-004-2020/UNAR-DISC-007-2020). Este comunicado trajo consigo opiniones varias dentro de la comunidad académica, sobre todo entre el gremio estudiantil, el cual, en principio, alegaba problemas de conectividad y acceso (y es que la modificación mostró, en cuestión de semanas, las verdaderas carencias del alumnado en estos términos, 
más que nada en su lugar de origen). Además, se revelaron las dificultades que los estudiantes con discapacidades podrían tener con el tránsito a la virtualidad, así como la crítica constante a que los programas de estudio no están diseñados para la modalidad virtual y a que el cambio repentino llevaría una marcada exclusión para aquellos educandos vulnerables.

Se podría creer que a los aprendices con dificultades de accesibilidad a Internet les es posible ingresar a un aula y hacer uso de la conexión para realizar y entregar trabajos, así como unirse a las sesiones sincrónicas u otras actividades curriculares, pero esto se esfumó con la circular UNA-R-DISC-027-2020 “Comunicación interna sobre la no presencialidad de estudiantes en las instalaciones de la UNA por el virus del covid-19". Aun así, por parte de la universidad, se desarrollaron iniciativas como dotar de chips de telecomunicaciones y de equipo tecnológico a esos estudiantes con condiciones de vulnerabilidad de acceso y conectividad.

La Vicerrectoría de Docencia de la Universidad Nacional realizó un estudio, denominado "Valoración de la experiencia de llevar a cabo los procesos educativos en la Universidad Nacional en presencialidad remota con apoyo tecnológico por parte de la población estudiantil. I período 2020", y entre los resultados más destacables están los siguientes:

Para analizar los resultados se han dividido los aspectos que se preguntaron en tres factores: institucionales, psicológicos y económicos-familiares. Los factores institucionales fueron los que más pesaron en el sentir de la población estudiantil, por ejemplo, la preocupación estudiantil en el rendimiento de los cursos $(72,8 \%)$, sintió ansiedad del cómo iba terminar el curso $(69,5 \%)$, se sintió desesperanzado por el sentimiento de incapacidad para hacer bien su trabajo (53\%). No obstante, también experimentaron aspectos positivos ya que el $57,1 \%$ se mostró optimista sobre las nuevas formas de trabajo y aprendizaje y el 51,3\% se siente sorprendido por los resultados inesperados de la experiencia.

Además, se le solicita a la población estudiantil que realicen una valoración general de la experiencia que ha tenido sobre las clases o actividades en entornos virtuales en comparación con los presenciales, al respecto el $54,0 \%$ considera que son inferiores a las clases presenciales, $38,1 \%$ manifiesta que son similares y el $7,9 \%$ que son superiores a las clases presenciales.

La valoración que hacen los estudiantes según el Campus en el que estudian, ubican a la población estudiantil del Campus Nicoya con una percepción más negativa sobre la enseñanza en los entornos virtuales, probablemente y como se ha demostrado en el transcurso del presente análisis, son los que han presentado condiciones más 
adversas en el I período lectivo 2020. No obstante, es importante que más de una tercera parte en todos los Campus considera que la enseñanza en entornos virtuales se asemeja a la enseñanza en presencialidad. (p. 61-62)

Las valoraciones y conclusiones anteriores abordan un panorama amplio o generalizado de toda la comunidad estudiantil universitaria. Ante esto, el presente trabajo recopila, mediante el instrumento de la encuesta, la opinión producto de una serie de interrogantes que se le plantean a 47 estudiantes de tercer y cuarto año de la carrera Gestión Empresarial del Turismo Sostenible. Ello pretende no solo confirmar lo concluido en el informe antes mencionado; además, aborda inconvenientes y dificultades en torno a cómo ha influido la conectividad a Internet en las clases, las sensasiones al respecto y cuál es la preferencia entre virtualidad y presencialidad.

Asimismo, este documento muestra tablas comparativas y gráficos, acordes con las preguntas y respuestas que los 47 estudiantes brindaron, según sus apreciaciones y criterios. Eso permite tener un acercamiento más específico y logra contextualizar una realidad para un grupo de alumnos determinado, a diferencia del informe, que tiene un enfoque mucho más general, en el escenario de la comunidad estudiantil, en términos globales, en la Universidad Nacional.

\section{Referentes conceptuales}

La conectividad, especialmente enfocada en el acceso a Internet, distribuida de manera igualitaria, equitativa y justa para todos, es fundamental si se desea tener una educación de calidad, aun cuando las metodologías cambian y se reajustan, de acuerdo con el contexto y las coyunturas en un momento determinado.

La presencialidad remota o educación a distancia no debe poner en entredicho la calidad de la educación, sino que, mediante esta, se debe velar por lograr una enseñanza y un aprendizaje significativos.

Se considera que la educación es relevante en la medida que promueve aprendizajes significativos para las personas como individuos y también como integrantes de una sociedad. El desarrollo humano sustentable debe apuntar precisamente a fortalecer esta doble contribución de los seres humanos. Para dicho fin, es imprescindible potenciar el desenvolvimiento de las capacidades, habilidades cognitivas y socioafectivas, ayudar a las personas a extender sus actitudes, aptitudes y conocimientos, orientándoles en una toma de decisiones respetuosa y solidaria (Quirós, 2019, p. 54).

Ante lo previo, es fundamental, en el marco de una pandemia y las restricciones que ella trae para la educación, encontrar las fórmulas, modelos y caminos que permitan a las partes involucradas optar por una formación de calidad. Bajo el 
contexto del covid-19, donde la presencialidad remota o educación a distancia es la única alternativa para que la educación continúe, resulta trascendental el acceso igualitario y equitativo al Internet, así como la buena conectividad, con el afán de que el ámbito educativo sea en verdad relevante y significativo para cada individuo y la sociedad.

Es a partir del Informe del Relator Especial de la ONU Frank La Rue (2011) que se considera el Internet como un derecho fundamental catalogado como de cuarta generación. Lo anterior, debido a que la conectividad le permite a la población no solo contar con libertad de opinión y expresión, sino que impulsa el progreso de la sociedad, ya que se convierte en un valioso instrumento para múltiples temas; en este caso, se recalca el de la educación y al respecto el informe menciona que el Internet:

Da acceso a una fuente de conocimientos amplia y en aumento, suplementa o transforma las formas tradicionales de enseñanza y, mediante iniciativas de "libre acceso", pone a disposición de la población de los Estados en desarrollo oportunidades de investigación académica antes inasequibles. Además, los beneficios educativos derivados del uso de Internet contribuyen directamente al capital humano de los Estados (p. 18).

El derecho a la conectividad se puede analizar, entonces, desde una índole social y debe ser satisfecho por parte del Estado; así lo menciona Bonilla (2016), al expresar que, considerándose un derecho de la sociedad, este debe "ser satisfecho con prestaciones públicas, al igual que el derecho a la educación, de la salud y previdencia social" (p. 9). Tal privilegio se ha convertido en una necesidad real de la población en cuanto a conocimiento, inclusión y participación, como consecuencia de la acelerada globalización y de los cambios tecnológicos que se viven con el paso del tiempo.

Es por lo dicho que "en varios países desarrollados se está examinando la posibilidad de que el suministro de banda ancha sea obligatorio en todos los hogares para evitar que algunos habitantes queden excluidos de las ventajas sociales y económicas que ofrece" (Ezenhfer, 2020, p. 67). Latinoamérica y sus países no deberían estar alejados de esta necesidad, la cual garantice la accesibilidad, conectividad y equidad a la sociedad en general, buscando, de una manera u otra, eliminar, al menos, cierto grado de desigualdad en este rubro.

La pandemia del Covid-19 sacó a la luz y "puso en evidencia un nuevo tipo de vulneración de derechos, como el del acceso a la conectividad. En la sociedad, quienes quedan excluidos de la red, no gozan de los ventajas sociales y económicas 
que ésta ofrece, como se aprecia en el caso puntual de la educación" (Ezenhfer, 2020, p. 67). Es por ello que esta crisis sanitaria, considerando las cosas positivas que podría dejar, mostró, inequívocamente, la disparidad entre personas y comunidades que no gozan de las mismas oportunidades respecto a quienes sí gozan de buena conectividad. Se revela, también, que la educación, como parte de su adaptación y cambio para incorporar la virtualidad, ha dejado a muchas personas en una posición desigual, complicada y con inconvenientes respecto a otros.

Por lo anterior, resulta necesario que la educación ponga en igualdad de condiciones a todos sus involucrados, tal como menciona Quirós (2019):

El enfoque de derechos humanos en la educación tiene como meta promover la inclusión sobre la base de que todas las personas son iguales en valor y dignidad. La entrada en vigor del derecho internacional sobre derechos humanos propicia que los sistemas educativos sean examinados, de modo que se eliminen los obstáculos en el acceso a la enseñanza y la inclusión educativa sea una realidad cada vez más palpable (p. 58).

Las instituciones, el Estado y la sociedad deben proveer condiciones, espacios y caminos que posibiliten, de modo imparcial, la accesibilidad a la educación, sin restricciones ni obstáculos que impidan garantizar el derecho a la educación de calidad, digna y con gran relevancia social. La Declaración Universal de los Derechos Humanos, en su artículo 26, expresa con claridad que "Toda persona tiene derecho a la educación" ya sea presencial, virtual, mixta o en cualquier otra modalidad.

\section{Metodología}

Esta investigación, es de carácter cualitativo, con un enfoque descriptivo, ya que buscó la percepción de 47 estudiantes de tercer y cuarto año de la carrera Gestión Empresarial del Turismo Sostenible, Campus Pérez Zeledón de la Universidad Nacional. Lo anterior se logró por medio de la aplicación de una encuesta constituida por 25 preguntas divididas en cuatro secciones:

- Sección 1: Aspectos generales de cada estudiante

- $\quad$ Sección 2: Las clases

- Sección 3: Los profesores

- $\quad$ Sección 4: La experiencia

En cada apartado, se realizaron interrogantes que permitieron describir lo que pensaba y sentía cada estudiante. La encuesta combinó preguntas abiertas y cerradas: las primeras recolectaron información que describía únicamente percepciones y 
sensaciones; las segundas recopilaron datos que tenían un estrecho vínculo con la comparación entre los modelos de enseñanza (presencial remota y presencialidad).

Los fundamentos para seleccionar la población estudiada se basaron en la capacidad que tenían los estudiantes de poder comparar, de forma más certera, la presencialidad con la virtualidad, ya que tenían al menos dos o más años de recibir clases presenciales y al menos un año en virtualidad, lo que generó mejores criterios comparativos respecto a un modelo y otro.

\section{Resultados}

De los 47 estudiantes encuestados, 30 viven en Pérez Zeledón o han estado recibiendo las clases allí, distribuidos a lo largo de los distritos del cantón. Los restantes 17 alumnos son de 6 diferentes cantones de la provincia de Puntarenas, Buenos Aires, Osa, Quepos, Parrita, Golfito y Coto Brus.

\section{Tabla 1}

Distribución geografica de los 47 estudiantes encuestados

\begin{tabular}{|c|c|c|c|}
\hline Estudiantes & Provincia & Cantón & Distrito \\
\hline 7 & & Buenos Aires & $\begin{array}{l}\text { Biolley, Boruca, Pilas, Buenos Aires, } \\
\text { Potrero Grande }\end{array}$ \\
\hline 2 & & Osa & Bahía Ballena \\
\hline 2 & Puntarenas & Coto Brus & Limoncito, Agua Buena \\
\hline 4 & & Quepos & Savegre, Naranjito \\
\hline 1 & & Parrita & Parrita \\
\hline 1 & & Golfito & Puerto Jiménez \\
\hline 30 & San José & Pérez Zeledón & $\begin{array}{l}\text { San Isidro, Daniel Flores, Rivas, La } \\
\text { Amistad, El General, Pejibaye }\end{array}$ \\
\hline
\end{tabular}

Nota: Elaboración propia.

La tabla anterior pretende destacar, en el contexto general, que una gran cantidad de los estudiantes vive en lo que se denomina zona rural, donde no se podría asegurar que la conexión a Internet es mala. Sin embargo, se puede percibir que no se tiene la misma velocidad que en el área urbana, como el caso de San Isidro, que cuenta con un alto grado tanto de urbanismo como de comercio y se podría decir que la calidad de la conectividad, independientemente de la empresa proveedora de red, es alta o buena.

De los 47 estudiantes encuestados, 22 indicaron que debieron desplazarse a un lugar distinto al de su residencia permanente, al momento de recibir su clase sincrónica. 


\section{Tabla 2}

Dificultades que han tenido en la presencialidad remota estudiantes quienes respondieron SÍ al desplazamiento a un lugar distinto al de su residencia

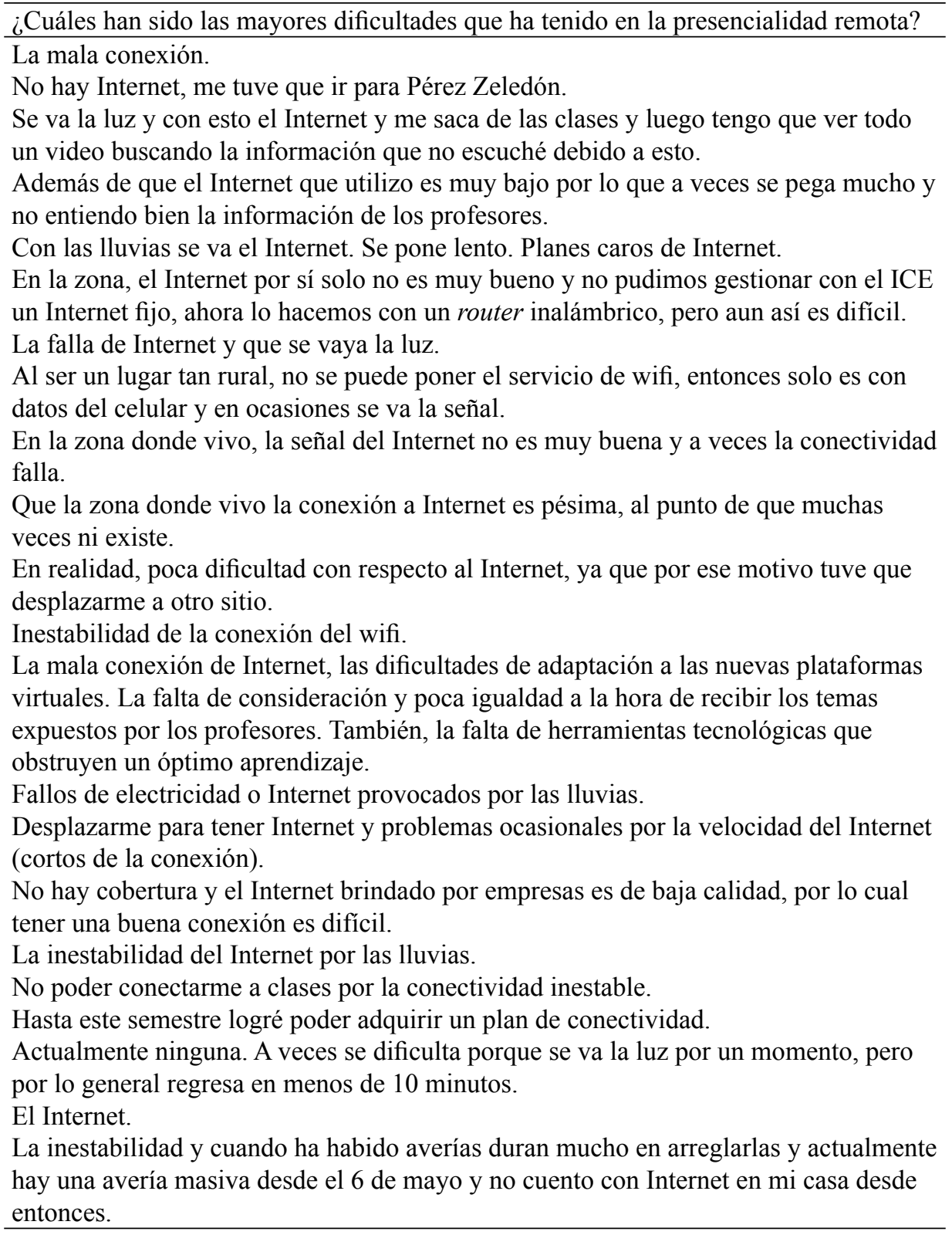

Nota: Elaboración propia. 


\section{Tabla 3}

Dificultades que han tenido en la presencialidad remota estudiantes que respondieron $\mathrm{NO}$ al desplazamiento a un lugar distinto al de su residencia

¿Cuáles han sido las mayores dificultades que ha tenido en la presencialidad remota? A veces hay problemas de audio o de imagen, por lo que no se puede llevar el hilo completo de la clase o, cuando llueve, se pone en mal estado la conexión.

Al tener la computadora y el celular conectado, el Internet no da abasto.

Cuando la velocidad del Internet es insuficiente o se va la luz.

El Internet de mi casa falla muy a menudo, ahora por las lluvias muchísimo más.

Ya coloqué Internet en la casa y ahora todo está mejor.

La conectividad a Internet.

Primeramente, que no cuento con una computadora propia y, por ende, dependo completamente de si la universidad puede o no prestarme una.

En Boruca se va mucho la electricidad y, por ende, dificulta bastante la conexión, la cual, además, se estropea fácil con el mal tiempo.

Internet deficiente y pérdida de luz en el hogar.

A la hora de estar en clases, cuando el profesor está explicando, el Internet en varias ocasiones se le va la señal, por lo cual me saca automáticamente de la reunión y no puedo entender la clase en su totalidad. En otras ocasiones, no puedo estar en la mayor parte de la clase, por lo cual tengo que volver a ver la clase grabada.

Cuando llueve el Internet es más lento.

Debido a la velocidad de conexión baja que presento, he tenido ciertos retrasos o caídas de Internet.

Algunas veces se va la conexión o se pone lenta.

El Internet es muy inestable.

Mala conexión.

Fallas en la electricidad.

Tener que poner Internet porque en mi casa no había y la señal es muy mala.

Los errores que se presentan en aquellas páginas o espacios habilitados para subir trabajos, exposiciones, exámenes, etc.

La baja velocidad del Internet y la avería de una computadora.

La calidad del Internet.

Afecta las lluvias para la señal de Internet.

Se va la electricidad y me quedo sin Internet, mayoritariamente los días de lluvias En tiempos de lluvias el Internet es muy inestable por la lentitud y que la señal se va más que todo cuando llueve algunas veces ha sido complicado.

Muy mala señal.

Que muchas veces me quedo sin señal cuando llueve.

Nota: Elaboración propia.

Las dos tablas anteriores muestran las respuestas de cada estudiante encuestado. De ellas se desprende que aquellos alumnos quienes no se desplazaron a un lugar 
diferente al de su residencia tienen casi las mismas dificultades que quienes sí se vieron en la necesidad de movilizarse. Ello indica que los que tomaron la decisión de moverse de sitio, con la finalidad de tener mejor conectividad durante sus clases y para entrega de trabajos, no lograron el objetivo esperado, en particular, en cuanto a las clases asincrónicas. Solamente dos estudiantes de los que se valieron del desplazamiento aseguraron no haber tenido problemas de conectividad en las sesiones sincrónicas, o bien en la entrega de trabajos en plataformas virtuales.

Se les consultó a los estudiantes cuáles habían sido los problemas más habituales a la hora de recibir las sesiones sincrónicas, que suponen ser el momento más importante de intercambio y construcción de saberes. Se sintetizó las respuestas y se tabularon en rubros.

\section{Tabla 4}

¿Qué problemas o inconvenientes ha tenido durante las sesiones sincrónicas?

\begin{tabular}{lc}
\hline \multicolumn{1}{c}{ Problema presentado } & $\begin{array}{c}\text { Cantidad de estudiantes con el } \\
\text { problema }\end{array}$ \\
\hline Equipo en mal estado & 1 \\
Equipo no adecuado & 2 \\
Espacio & 1 \\
Mala conexión & 31 \\
Se va la luz & 8 \\
Ninguno & 4 \\
\hline
\end{tabular}

Nota: Elaboración propia.

De los cuatro que indicaron que no tuvieron ningún problema en las sesiones sincrónicas, dos fueron quienes se desplazaron, en busca de mejor conectividad, a un lugar diferente al de su residencia y los otros dos viven en San Isidro. Esto puede ser una señal de que, al ser el casco urbano del cantón de Pérez Zeledón, se tienen mejores condiciones de conectividad.

Es claro que la mala conexión se convierte en el problema predominante entre los estudiantes. Esto les causa dificultades e inconvenientes, no solamente en las sesiones sincrónicas, sino también en el acceso a las plataformas dadas por los profesores. Se resalta, de forma mayoritaria, el Aula Virtual Institucional, que no les permite subir los trabajos, tareas, foros y diferentes asignaciones, debido a la baja calidad de la conexión. 
Los cortes en el fluido eléctrico son una de las razones por las cuales se pierde la conexión y, en gran medida, se entrelazan la falta de energía eléctrica y la mala conexión con las fuertes lluvias generadas en la época lluviosa. A esto se suma que esta última es la estación climatológica más larga del año y resulta que coincide con el ciclo lectivo.

\section{Aprendizaje: virtualidad o presencialidad}

\section{Gráfico 1}

Comparativa del aprendizaje respecto a la presencialidad

\section{En comparación con la presencialidad, ¿cree que el aprendizaje es el mismo con la virtualidad, es mejor o no aprendo igual?}

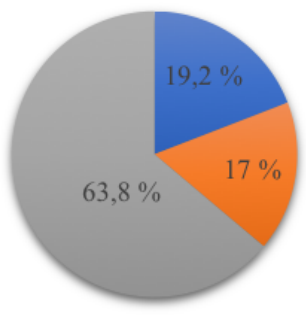

Es lo mismo

- Es mejor la virtualidad para aprender

Es peor la virtualidad para aprender

Nota: Elaboración propia.

Del gráfico anterior, es importante mencionar que los 30 estudiantes quienes consideran que la virtualidad es peor para aprender coinciden en su totalidad con todos los que tuvieron problemas de mala conexión. Se podría entender, entonces, que la conectividad deficiente genera malas sensaciones en relación con el aprendizaje.

Incluso, la carga excesiva de trabajos y actividades extraclase puede ser un aliciente para sentirse disconforme con la virtualidad en el aprendizaje, lo cual se refleja en el siguiente gráfico: 


\section{Gráfico 2}

Comparativa de la cantidad de trabajos y asignaciones entre la virtualidad y presencialidad

En comparación con la presencialidad, ¿cree que los trabajos, tareas y actividades extra clase son mas, iguales o menos, con respecto a la virtualidad?

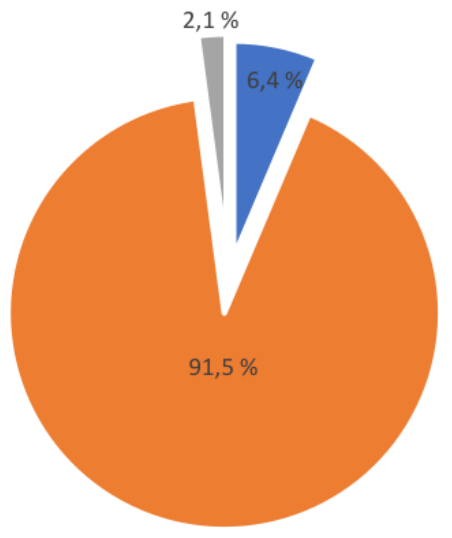

- Igual cant idad de trabajos, tareas y actividades

- Más cantidad de trabajos, tareas y actividades

- Menos cantidad de trabajos, ta reas y actividades

Nota: Elaboración propia.

Lo anterior evidencia que la mala conexión a Internet y las dificultades que esto trae consigo, así como una mayor carga de trabajos, tareas y actividades extra-clase, generan un potencial rechazo a la virtualidad, en términos generales. Inclusive, los estudiantes fueron consultados sobre si creían que existe relación entre la cantidad de trabajos y el nivel de aprendizaje y un $58 \%$ indicó que no consideraba positivo el efecto de tener más trabajos y tareas para un mayor aprendizaje. Y es que

La virtualidad se asocia, de forma recurrente, con un incremento de la carga lectiva, aspecto que denota la necesidad de revisar los procesos formativos y establecer indicadores y parámetros eficaces en este ámbito, con el objetivo de no saturar al alumnado de actividades extracurriculares que pueden ocasionar la deserción del mismo (Tejedor, Cervi, Tusa y Parola, 2020, p. 14).

Es importante mencionar que, de los 47 estudiantes, 45 aseguraron que la conectividad y la virtualidad han influido de manera negativa en su carrera profesional, promoviendo condiciones como frustración, cansancio, pérdida de lecciones y hasta problemas de salud como dolor del túnel carpiano, pérdida de visión y molestias de 
espalda. Es común escuchar, entonces, casi de forma universal, el comentario de que "el paso de la presencialidad a la virtualidad ha sido considerado por la mayoría del alumnado como cambio negativo" (Tejedor, Cervi, Tusa y Parola, 2020, p. 7).

Los profesores, si bien no tienen control sobre la conectividad ni las dificultades de los estudiantes, juegan un papel sumamente trascendental en todo el proceso. Se les confiere la tarea de ser motivadores y cautivar a los estudiantes mediante el proceso de enseñanza y aprendizaje. Ellos también tienen su dosis de inconvenientes, los cuales no se abordan en este trabajo; no obstante, son un tema de relevancia por analizar en su debido momento.

A los estudiantes se les consultó si consideraban que las clases en presencialidad remota eran más aburridas, más entretenidas o iguales a las de la presencialidad y ello se compara con el deseo que ellos mismos tienen de volver a esta, quedarse en la virtualidad, o bien una combinación de ambas.

\section{Tabla 5}

Percepción del estudiante de la clase y preferencias entre virtualidad y presencialidad

\begin{tabular}{|l|l|l|l|}
\hline Más aburridas & 28 & \multicolumn{2}{|c|}{} \\
\hline Más entretenidas & 9 & \multicolumn{2}{|c|}{} \\
\hline $\begin{array}{l}\text { Iguales a las de la } \\
\text { presencialidad }\end{array}$ & 10 & & \\
\hline \multirow{5}{*}{} & & Prefiero volver a la presencialidad & 16 \\
\cline { 2 - 4 } & & Prefiero seguir en la virtualidad & 7 \\
\cline { 2 - 4 } & & Mixto & 24 \\
\hline
\end{tabular}

Nota: Elaboración propia.

Es interesante que, a pesar de que los estudiantes consideran más aburridas las clases en virtualidad, no representan la mayoría quienes desean volver a la presencialidad. Aun con las vicisitudes que tienen, resaltan que un modelo mixto tiene sus beneficios. Esto podría entenderse como que, de alguna manera, el alumnado comprende que las herramientas tecnológicas y la virtualidad tienen aspectos positivos rescatables y merecedores de una adecuada implementación.

Ante lo anterior, se preguntó a los estudiantes: ¿Consideran que los profesores están preparados para dar clases en virtualidad? Un $67 \%$ de ellos indica que sí, un $15 \%$ que no y el grupo restante manifiesta que tal vez. Esto da a entender que los educandos, más allá de todas las dificultades del proceso, consideran, mayoritariamente, que los docentes logran prepararse para impartir un curso de 
la mejor manera, a pesar de sus limitaciones tecnológicas y complejidades. Sin embargo, cargan el peso de que los contenidos no se transforman adecuadamente a la virtualidad, lo que produce que, en algunos casos, el alumno considere que la lección se torna más aburrida (ver tabla 5).

En su mayoría, los profesores se encontraron de golpe con un cambio en el método para impartir clases, lo que los sacó, de manera repentina y abrupta, de su zona de confort. Fueron sorprendidos con una nueva metodología, llena de retos, dificultades en todas direcciones y factores que salen de su control, que llegan a repercutir de algún modo en el estudiante.

Las dificultades evidenciadas en la modalidad de enseñanza implementada en las aulas virtuales se producen en diferentes momentos de la intervención docente:

En la planificación de la clase: cuando el profesor no pondera la cantidad de actividades propuestas a los alumnos en una clase y genera una demanda excesiva de producciones que abruma a los estudiantes.

En la gestión de la clase: cuando el docente no establece una articulación entre las actividades asincrónicas y las sincrónicas y se evidencia una brecha entre los diferentes tipos de actividades propuestas.

En el cierre de la clase: cuando se omite una actividad evaluativa que permita al estudiante expresar aquello que aún no ha logrado aprender y que desea conocer. (Vicenzi, 2020, p. 5)

A pesar de todo, el estudiante es consciente de que la presencialidad remota ha traído consigo un cambio total en el paradigma de la enseñanza y el aprendizaje. Se genera, así, una adaptación, bajo la existencia de rangos aceptables y medidas controlables que asumen tanto el profesor como el alumno, en el constante desarrollo de los cursos y clases.

\section{Conclusiones}

La conectividad como derecho humano y la pandemia como un fenómeno de cambio han descubierto la verdadera cara de la realidad que viven los estudiantes, en este caso, de la carrera de Gestión Empresarial del Turismo Sostenible. Se muestra que la conectividad no solamente se trata de tener conexión y ya, sino, más bien, de que esta sea de calidad, veloz y estable. Sin embargo, "solo la buena interacción alcanzada en la sociedad civilizada y un Estado fuerte podrá resistir el aislamiento social que vulnera una serie de derechos. Asimismo, solo una educación 
democrática en todos los niveles podrá afrontar el futuro apocalíptico al cual se expone la humanidad" (Huanca, Supo, Sucari y Supo, 2020, p. 125).

La virtualidad llegó para quedarse, mas no para reemplazar la presencialidad; busca ser un apoyo al docente en los procesos de formación. Y es que, definitivamente, al instruir en la actualidad, omitir la tecnología como parte del proceso de enseñanza sería una gran pérdida para el procedimiento formativo, en ambos sentidos, ya que tanto el estudiante como el profesor estarían desaprovechando una gran herramienta metodológica.

El desafío que se potencia hacia una modalidad híbrida de la educación supone dejar atrás las modalidades formativas centradas en la divulgación de conocimientos y abrazar estrategias de enseñanza centradas en el diseño de experiencias de aprendizaje. Se aspira a una organización de los contenidos mediante una narrativa multimedia, gestionada a través de una secuencia de aprendizaje que jerarquice el trabajo colaborativo y la conformación de una comunidad de aprendizaje (Vicenzi, 2020, p. 7).

Para el estudiante, es fundamental que el docente logre encontrar metodologías didácticas y pedagógicas adecuadas, las cuales se adapten más allá de las dificultades que se presentan con la conectividad. Se pretende encontrar, así, los espacios oportunos para aprender, aun por encima de los inconvenientes que se enfrentan, con el afán lograr el objetivo de culminar su carrera universitaria de forma exitosa.

Es importante resaltar la voluntad de los estudiantes quienes, a pesar de lo que se podría catalogar como una "experiencia generalmente frustrante y cansada", siguen dispuestos a aprender, mejorar y continuar con su carrera profesional. Esto aplica, sobre todo, en los alumnos de tercer y cuarto año de la carrera Gestión Empresarial de Turismo Sostenible, Sede Regional Brunca, Universidad Nacional, que se encuentran en la ruta final de su profesión, lo cual hace que prevalezca el esfuerzo, el interés y los deseos de culminar, de la mejor manera, sus estudios, más allá de lo retador del proceso. 


\section{Referencias}

Bonilla, H. M. (2016). El acceso a Internet como derecho fundamental. Revista jurídica IUS Doctrina, 9(15). https://revistas.ucr.ac.cr/index.php/iusdoctrina/article/view/27476

Departamento de Registro. (2020) Valoración de la experiencia de llevar a cabo procesos educativos en la Universidad Nacional en presencialidad remota con apoyo tecnológico por parte de la población estudiantil. I periodo 2020. https:// www.registro.una.ac.cr/see/index.php/perfil-del-estudiante-primer-ingreso 2018/ valoracion-de-la-experiencia-estudiantes

Enzenhofer, P. (2020). Educación en red en tiempos de pandemia. La conectividad como derecho humano. XII Congreso Internacional de Investigación y Práctica Profesional en Psicología. XXVII Jornadas de Investigación. XVI Encuentro de Investigadores en Psicología del MERCOSUR. II Encuentro de Investigación de Terapia Ocupacional. II Encuentro de Musicoterapia. Facultad de Psicología, Universidad de Buenos Aires, Buenos Aires.

Huanca-Arohuanca, J., Supo-Condori, F., Sucari-León, R. y Supo-Quispe, L. (2020). El problema social de la educación virtual universitaria en tiempos de pandemia, Perú. Innovaciones Educativas, 22(especial), 115-128. https://doi.org/10.22458/ ie.v22iEspecial.3218

Naciones Unidas. (1948). Declaración Universal de los Derechos Humanos. http://www. un.org/es/universal-declaration-human-rights/

Organización de Naciones Unidas. Asamblea General. (2011). Informe del Relator Especial sobre la promoción y protección del derecho a la libertad de opinión y expresión Frank Le Rue./HRC/17/27 del 16 de mayo del 2011. https://www.acnur.org/fileadmin/ Documentos/BDL/2015/10048.pdf?view=1

Quirós-Vargas, E. (2019). Derechos Humanos y Realidad Educativa. Revista Latinoamericana de Derechos Humanos, 30(1), 47-60. https://doi.org/10.15359/rldh.30-1.2

Tejedor, S., Cervi, L., Tusa, F. y Parola, A. (2020). Educación en tiempos de pandemia: reflexiones de alumnos y profesores sobre la enseñanza virtual universitaria en España, Italia y Ecuador. Revista Latina de Comunicación Social, 78, 1-21. https://www.doi. org/10.4185/RLCS-2020-1466

Vincenzi, A. D. (2020). Del aula presencial al aula virtual universitaria en contexto de pandemia de COVID-19. Avances de una experiencia universitaria en carreras presenciales adaptadas a la modalidad virtual. Debate Universitario, 8(16). http://portalreviscien. uai.edu.ar/ojs/index.php/debate-universitario/article/view/238 
